\title{
Case report description of a collaborative approach to thoracic duct embolization in patients with congenital heart disease
}

\author{
Mitchell A Luangrath ${ }^{1}$, Jason Pinchot ${ }^{2}$ and Luke J. Lamers ${ }^{3 *}$
}

\begin{abstract}
Background: Medical management and surgical interventions for the lymphatic disorders chylothorax and plastic bronchitis are often unsuccessful. Single center data suggest that thoracic duct embolization is a more efficacious treatment for refractory lymphatic disorders, yet these outcomes have not been replicated.

Case Presentation: This case series describes a collaborative approach to the development of a successful lymphatic intervention program for congenital heart disease patients with persistent lymphatic disorders and defines a potential anatomic contraindication.

Conclusions: Successful lymphatic interventions can be performed at institutions with experienced providers working closely together to treat patients with complex congenital heart disease. Proper patient selection is essential and based on our results the presence of extensive lower body venous occlusion may be a contraindication and should be thoroughly investigated prior to attempts at thoracic duct embolization.
\end{abstract}

Keywords: Chylothorax, Plastic bronchitis, Thoracic duct embolization

\section{Background}

Percutaneous thoracic duct (TD) embolization is a minimally invasive therapy for the treatment of chylothorax and extensive interventional radiology literature exists documenting procedural indications, techniques and outcomes in adults $[1,2]$. The incidence of chylothorax in pediatrics is small, yet for patients with congenital heart disease (CHD) postoperative chylothorax has been reported to occur between 2 and 5\% and is associated with significant morbidity and mortality [3]. Conservative management often fails and invasive surgical therapies have variable success rates and documented complications [3-5]. Recently, TD embolization has been applied to CHD patients and been shown to be more efficacious than conventional therapies for refractory chylothorax and plastic bronchitis [6-8]. These two disease processes are believed to have similar etiologies related to abnormalities in the lymphatic system anatomy and drainage. The majority of knowledge about the

\footnotetext{
* Correspondence: llamers@pediatrics.wisc.edu

${ }^{3}$ Division of Pediatric Cardiology, Department of Pediatrics, University of Wisconsin School of Medicine and Public Health, 600 Highland Ave, Madison, WI 53792-4108, USA

Full list of author information is available at the end of the article
}

lymphatic system and TD embolization in pediatrics can be attributed to work from a single center [6-9] that has greatly expanded our understanding and holds promise for future therapies.

TD embolization in CHD patients is a complicated intervention requiring a collaborative approach. Lymphangiography and percutaneous transabdominal thoracic duct access are advanced interventional radiology techniques and the intricacies of palliated CHD require experienced cardiology input. At our institution, a robust adult interventional radiology program exists with experience in TD embolization and there is a growing population of CHD patients who may benefit from this therapy locally. Thus, the aim of this case series is to describe the preliminary results of our collaborative approach to the development of a $\mathrm{CHD}$ focused lymphatic interventional program.

\section{Case Presentation \\ Case \#1}

The initial TD embolization occurred in a 4 year old $15 \mathrm{Kg}$ male with situs inversus, D-looped ventricles, D-malposed great vessels with pulmonary atresia (I,D,D), ventricular septal defect, transitional atrioventricular canal. $\mathrm{He}$ 
previously underwent a central shunt and left-sided bidirectional Glenn. His surgical repair consisted of mitral valve cleft closure, hemi-Mustard baffling of the inferior vena cava to the right sided atrium and Rastelli type VSD closure with a $17 \mathrm{~mm}$ right ventricle to pulmonary artery conduit. Post-operative course was complicated by prolonged chylothorax. Chest tube (CT) output averaged $33 \mathrm{ml} / \mathrm{kg} /$ day for the week prior to TD embolization which was done on post-operative day (POD) \#25 without pre-procedure lymphatic magnetic resonance (MR) imaging.

The intervention began with cardiac catheterization assessment of hemodynamics and imaging to rule out intracardiac shunts. Lymphangiography was then performed as previously described [1] via ultrasound guided access of bilateral inguinal lymph nodes with 22-gauge spinal needles and injection of $2.5 \mathrm{~cm}^{3}$ of ethiodal allowing visualization of the cisterna chyli that was accessed via a transabdominal puncture with a 22-guage Chiba needle and V18 wire. A Rapid Transit micro catheter was positioned into the TD and iodinated contrast was injected to perform detailed lymphangiography. The mid to lower TD was embolized with Tornado and Hilal microcoils and approximately $2 \mathrm{~cm}^{3}$ of a 1:1 mixture of n-butyl cyanoacrylate (n-BCA) glue and ethiodol. Two days post TD embolization the chest tubes were removed and the patient was discharged home 5 days post intervention with no complications and no chylothorax recurrence.

\section{Case \#2}

A 5 year old $19 \mathrm{Kg}$ male with hypoplastic left heart syndrome (HLHS) palliated through fenestrated Fontan presented to our institution with a 1 year history of plastic bronchitis. Medical management was not providing clinical benefit and a pre-procedure MR lymphangiogram following protocols defined by Dori et al. $[6,9]$ demonstrated abnormal lymphatic connections from the TD to the right bronchus (Fig. 1).

Given a known history of innominate vein occlusion, documented veno-venous collaterals from the left subclavian vein to the pulmonary veins and a patent Fontan fenestration there was concern for potential systemic embolization of ethiodol. Thus, the procedure began with cardiac catheterization and temporary balloon occlusion of the Fontan fenestration and via left basilic vein access a $4 \mathrm{~mm}$ balloon was positioned in the subclavian vein to temporarily occlude numerous small veno-venous collaterals. Lymphangiography was then performed via bilateral inguinal lymph node injections of $4.5 \mathrm{~cm}^{3}$ of ethiodal and the cisterna chyli and subdiaphragmatic portion of the TD were accessed with a V18 wire and 3-French AccuStick sheath. The TD was highly tortuous at the diaphragm with a branch from the superior aspect connecting directly to the right bronchus, similar to pre-procedure MR-lymphangiography

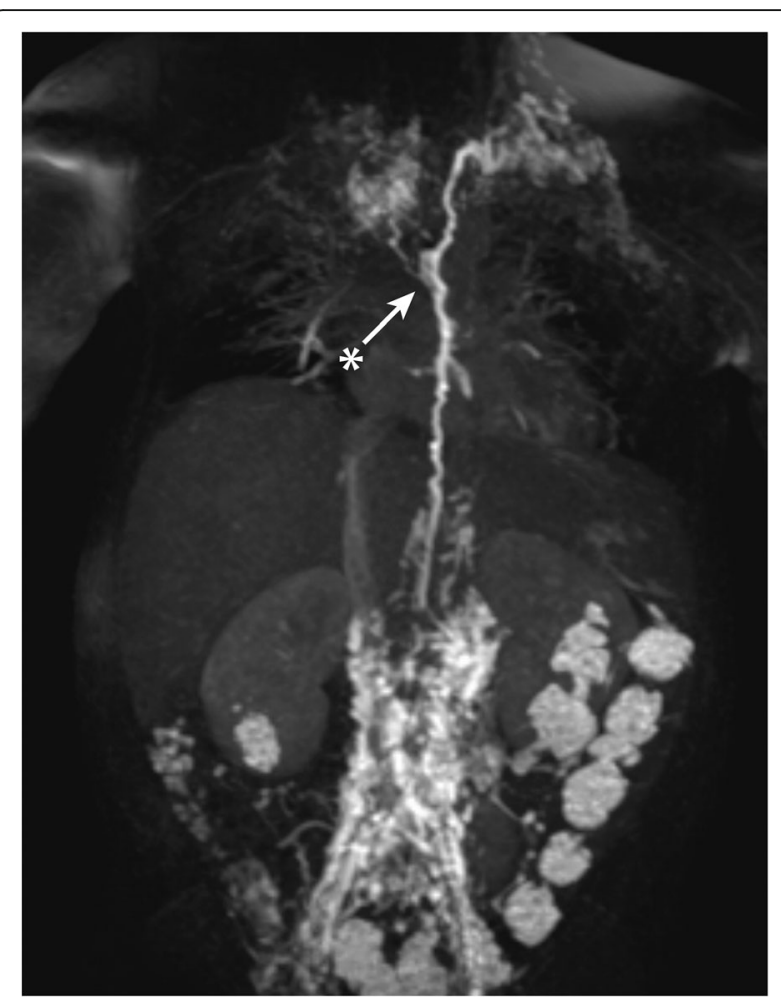

Fig. 1 Pre-procedure MR lymphangiography demonstrating TD drainage to a diffuse network of small lymphatic channels near the left side of the innominate vein. * identifies abnormal lympho-pulmonary connections to the right upper lung which was the location of recurrent casts in this patient with plastic bronchitis

(Fig. 2). Due to the tortuous nature of the TD, attempts at advancement of a V18 wire into the thoracic portion of the TD was difficult resulting in loss of access to the TD by which time the ethiodol had emptied from the cisterna chyli and land marks for repeat TD access were lost; therefore, the procedure was concluded. As has been reported with ethiodal lymphangiography alone and with TD interruption from recurrent needle trauma [2], following this procedure, the patient had no cast production for 2 months. He was weaned from daily inhaled alteplase and shortly thereafter developed recurrent casts; therefore, he was brought back for repeat attempt at TD embolization.

With the second procedure, the Fontan fenestration and the left subclavian vein were again temporarily balloon occluded and the cisterna chyli was accessed following opacification with $4 \mathrm{~cm}^{3}$ of ethiodal. Given the known tortuosity of the TD, a microcatheter was positioned just below the diaphragm and an 0.014 transcend wire was manipulated through the tortuous portion of the TD. The previously imaged connection from the TD to the right bronchus was no longer visible. The TD was embolized with multiple Nester and tornado coils and glued with $3 \mathrm{~cm}^{3}$ of a $3: 1$ solution of 


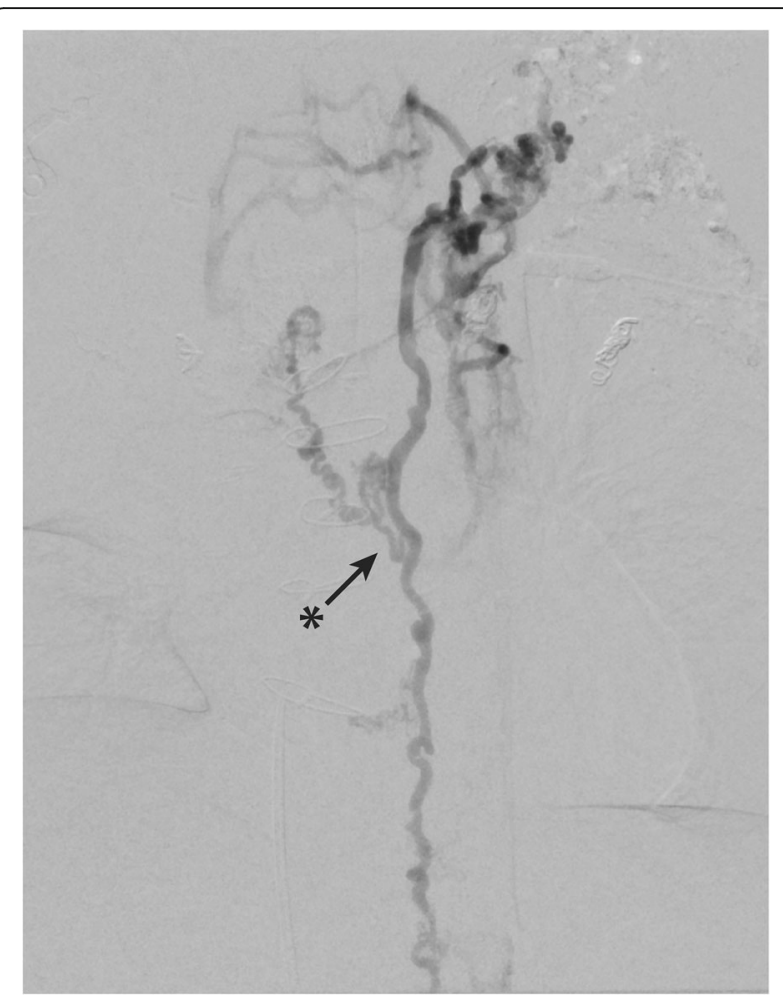

Fig. 2 Pre TD embolization in patient \#2. Pre TD embolization imaging confirms MR lymphangiography findings * of abnormal lymphatic connections to right upper lung segment

Ethiodol and n-BCA glue (Fig. 3). The patient is now 8 months post TD embolization, has had no subsequent casts and has been weaned off of all inhaled therapies.

\section{Case \#3}

A 6 month old $7 \mathrm{Kg}$ male with nasal pyriform aperture stenosis was hospitalized following birth due to CHD consisting of double outlet right ventricle with valvar and branch pulmonary artery (PA) stenosis required left PA plasty and BTT shunt as infant and tracheostomy for recurrent failed extubation at 6 weeks of age. At 4 months he underwent a complete cardiac repair. Postoperative course was complicated by mediastinitis and prolonged chylothorax not responsive to conservative management and an attempt at laparoscopic TD ligation on POD \# 27. CT output averaged $50 \mathrm{~cm}^{3} / \mathrm{kg} /$ day for the week prior to TD embolization on POD \#43. Preprocedure MR-lymphangiography demonstrated normal TD anatomy with drainage to the $L$ subclavian vein with suggestion of a chylous leak into the $\mathrm{L}$ pleural space along with chylo-lymphatic reflux in the upper abdomen suggesting elevated central lymphatic pressures (Fig. 4).

Given concerns for systemic embolization of ethiodol in the presence of a residual atrial septal defect (ASD) with elevated filling pressures, it was decided to perform ASD device closure prior to TD embolization. Ultrasound and

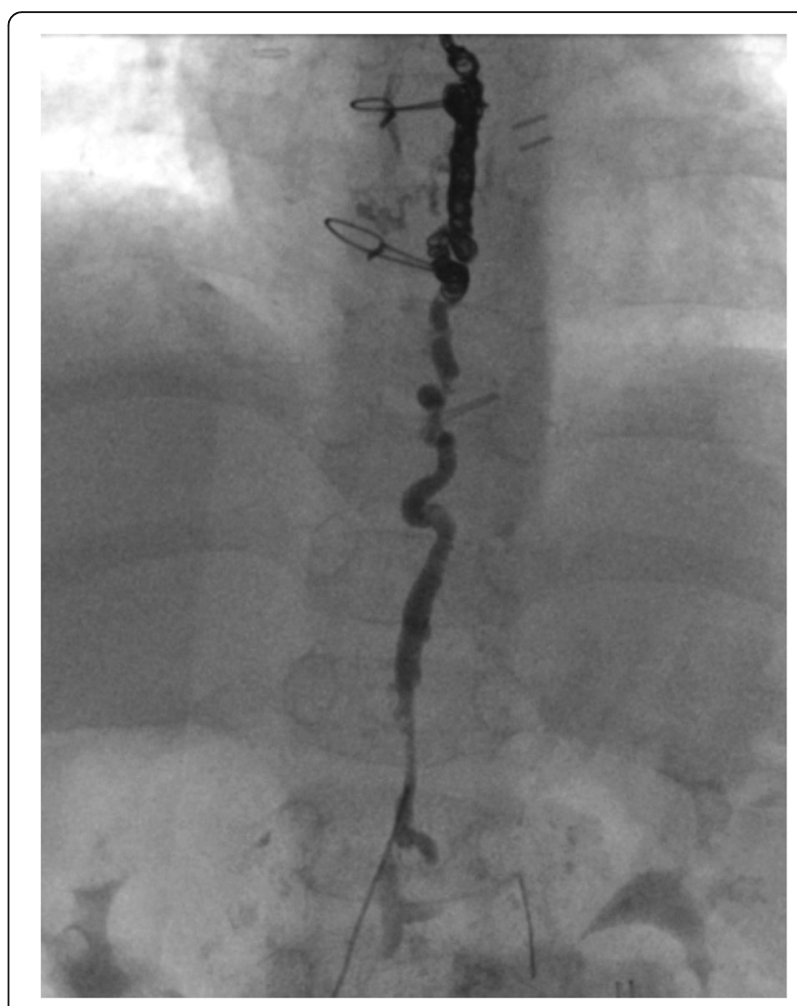

Fig. 3 Post intervention demonstrates coils and contrast occluding the TD

contrast imaging during the procedure documented bilateral femoral venous occlusion extending up the inferior vena cava to the level of the renal veins. Transesophageal echo imaging demonstrated a $4 \mathrm{~mm}$ central ASD. The left atrial pressure was $12 \mathrm{mmHg}$ and the right atrial pressure was $10 \mathrm{mmHg}$ and the ASD was closed with a $4 \mathrm{~mm}$ Amplatzer septal occluder delivered by trans-hepatic access. The patient then underwent successful lymphangiography (Fig. 5) confirming TD anatomy seen on preprocedure MR. TD embolization was performed from the mid TD within the chest to below the cisterna chyle with similar technique as described above using multiple Concerto and Nester coils as the scaffold for $4 \mathrm{~cm}^{3}$ of $3: 1$ ethiodol to nBCA glue solution (Fig. 6).

Post procedure course initially demonstrated a trend down in CT output with simultaneous development of abdominal distension and ascites. On POD \#9 a peritoneal drain was required due to respiratory compromise from abdominal distension. The peritoneal fluid was chylous ascites and the peritoneal drain remained in place for 3 weeks. CT output never dramatically improve and the CT remained in place for 2 months post TD embolization. X-rays up to 6 weeks post TD embolization demonstrated limited clearance of ethiodol from the abdomen and thorax with persistent effusions in multiple body compartments. Eventually with diligent medical management all 


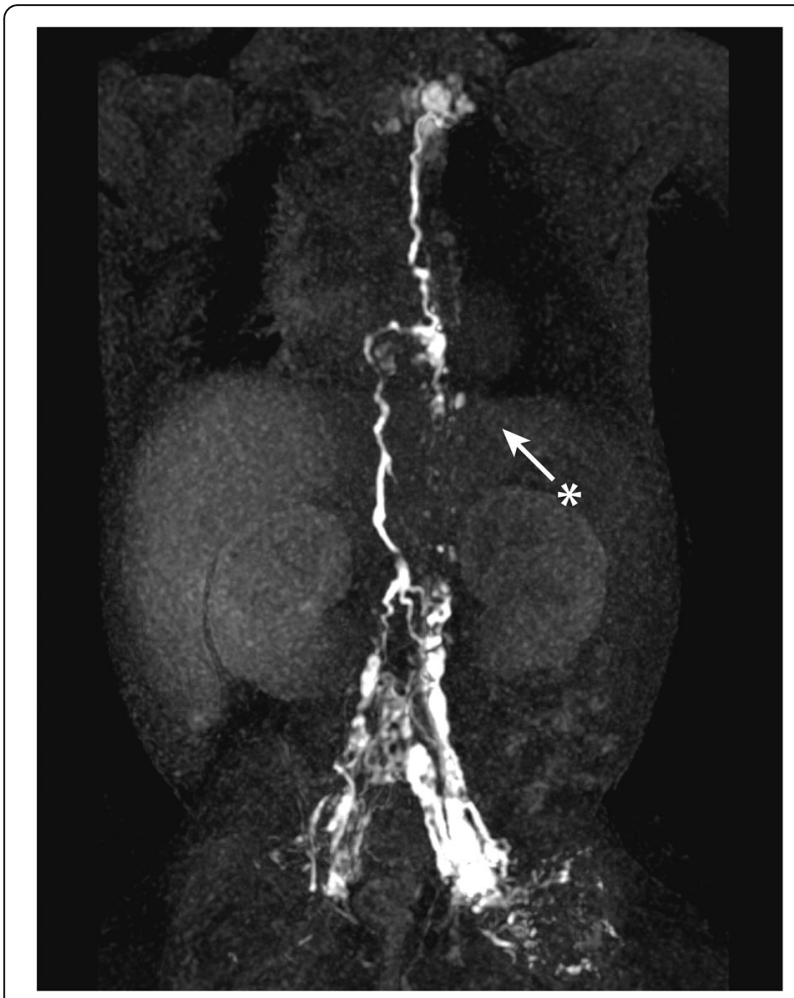

Fig. 4 Pre-procedure MR lymphangiography demonstrating TD drainage to the left side of the innominate vein. ${ }^{*}$ identifies abnormal lymphatic connections to the left pleural space, side of recurrent postoperative chylous effusion

drains were removed and feeds (Lipistart) were slowly advanced to full and the patient was discharged to home at 9 months of age, nearly 4 months post TD embolization.

\section{Discussion}

Thoracic duct embolization, first described for treatment of high output chylothorax in adults [1, 2], is now a viable therapy for treatment of refractory lymphatic abnormalities in pediatric CHD patients that appears to be more efficacious than conservative management or surgical intervention $[7,8]$. This case series describes our initial experience applying procedural details first described in the late 1990s that have recently been used in patients with $\mathrm{CHD}$ at a single institution [6-8]. Our aim was to demonstrate through collaboration between interventional radiology and pediatric cardiology that new centers can safely and effectively perform lymphatic interventions.

Our preliminary success can be attributed to two factors and should be replicable at institutions with the appropriate providers. First, our adult interventional radiologists have extensive experience with TD embolization and the technique is similar in adults and children. There may be anatomic and physiologic advantages leading to higher success rates in pediatric CHD patients who often have dilated cisterna chyle and TD secondary to elevated central

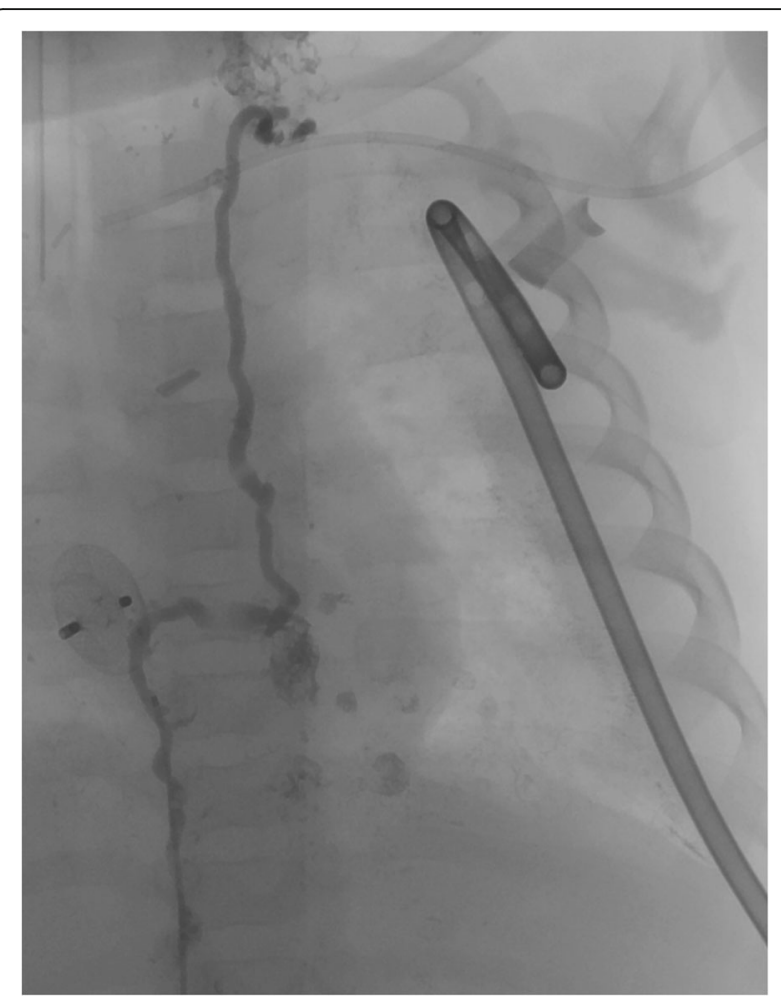

Fig. 5 Pre TD embolization in patient \#3. Pre TD embolization imaging confirms MR lymphangiography with lymphatic drainage into left pleural space

venous pressures along with thinner abdomens making transabdominal access of the TD more likely to be successful. Itkin et al. [2] in the largest adult series to date performing TD embolization was able to cannulate the TD in $67 \%$ of patients (73 of 109) compared to greater than $94 \%$ successful rates in two recent pediatric case series $[8,9]$. The second factor leading to success is that Dori and colleagues at the Children's Hospital of Philadelphia have thoroughly described pre-procedure MR imaging protocols, procedural intranodal lymphangiography and embolization techniques providing a detailed guide for program initiation [6-9]. This group's most recent publication further expands the understanding of the lymphatic system by identifying three distinct etiologies of chylothorax in CHD patients [6]. Unlike in adults, traumatic leak from the TD is relatively uncommon in children (8\%). More often chylothorax is associated with pulmonary lymphatic perfusion syndrome (PLPS) where lymph flows from the TD towards the lungs through multiple abnormal lymphatic connections in the chest (56\%) or central lymphatic flow disorder (CLFD) which is a condition with abnormal reduced or absent central lymphatic flow, effusions in more than one body compartment and the presence of dermal backflow through lymphatic collaterals into the abdominal wall (36\%). The differentiation of chylothorax etiology through MR lymphangiography is 


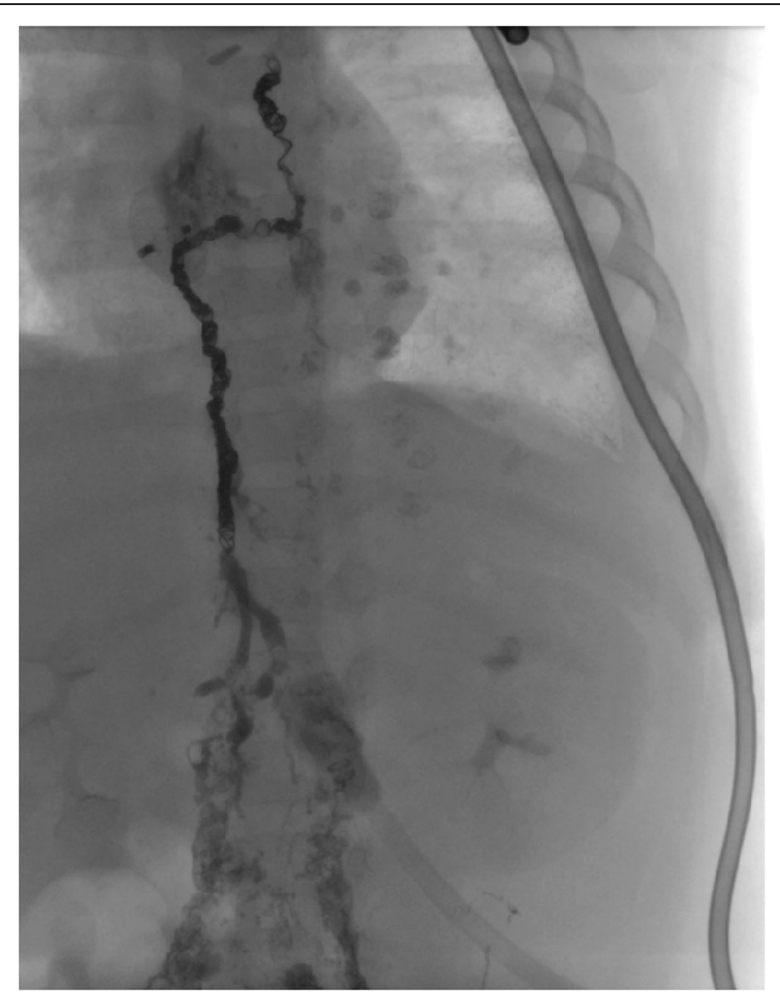

Fig. 6 Post intervention demonstrates coils and contrast occluding the TD

imperative as patients with TD leak or PLPS typically have resolution of symptoms following successful TD embolization whereas the diagnosis of CLFD at the current time appears to be uniformly fatal.

Our first two interventions demonstrate the clinical benefits of TD embolization. Both patients had failed conventional therapies and following TD embolization a postoperative chylothorax completely resolved within $48 \mathrm{~h}$ and the second patient has had resolution of plastic bronchitis for 8 months. Our third TD embolization demonstrates an unknown of lymphatic interventions. Preprocedure MR lymphangiography demonstrated normal TD drainage to the L subclavian vein with what was interpreted as a lymphatic leak into the L pleural space. Based on published data, one would have expected resolution of the chylothorax following successful TD embolization with this lymphatic anatomy [6]. In this patient, imaging in preparation for ASD closure demonstrated extensive lower body central venous occlusion. A yet to be determined and key detail of TD embolization is what happens to the lower body lymphatic drainage post TD embolization. It is presumed that over time new lymphovenous connections develop that bypass the occluded TD. Early studies demonstrate the presence of lymphovenous shunts in man and lack of lymphovenous communications are associated with lymphedema in certain clinical scenarios [10, 11]. Also there is increasing success with microsurgical creation of lymphovenous shunts as a therapy for systemic lymphedema [12]. We propose that given the lack of normal venous return from the lower body there was an inability to develop lymphovenous connections capable of decompressing TD backflow. Based on this single complicated case, the diagnosis of significant lower body major vessel venous occlusion may be a contraindication and should be ruled out in advance of attempts at TD embolization.

\section{Conclusion}

Here we report the initial results of our collaborative lymphatic intervention program in CHD patients. To our knowledge, these cases represent the first percutaneous TD embolizations performed in pediatric CHD patients outside the single center responsible for all previously published literature. This work suggests that lymphatic interventions can be conducted at institutions with experienced providers working closely together to care for complex CHD patients. Proper patient selection is key and based on our results, the presence of extensive lower body venous occlusion may be a contraindication and should be investigated prior to consideration of TD embolization.

\section{Abbreviations}

ASD: Atrial septal defect; BTT: Blalock Thomas Taussig; CHD: Congenital heart disease; CLFD: Central lymphatic flow disorder; CT: Chest tube;

HLHS: Hypoplastic left heart syndrome; MR: Magnetic resonance; n-BCA: n-butyl cyanoacrylate; PA: Pulmonary artery; PLPS: Pulmonary lymphatic perfusion syndrome; POD: Post operative day; TD: Thoracic duct

\section{Funding}

The authors declare that there is no funding associated with this case series.

\section{Availability of data and materials}

Data sharing not applicable to this article as no datasets were generated or analyzed during the current study.

\section{Authors' contributions}

$M L$ carried out the clinical chart review and drafted the manuscript. JP and $\mathrm{LL}$ conceived the case series, collaborated to perform all procedures described and help draft the final manuscript. All authors have read and approved the final manuscript.

\section{Ethics approval and consent to participate}

This study was approved by the University of Wisconsin Institutional Review Board. Consent to participate obtained from parent or legal guardian of the children described in this case series.

\section{Consent for publication}

Consent to publish de-identified data obtained from parent or legal guardian of the children described in this case series.

\section{Competing interests}

The authors declare that they have no competing interests.

\section{Publisher's Note}

Springer Nature remains neutral with regard to jurisdictional claims in published maps and institutional affiliations. 


\section{Author details}

'Department of Pediatrics, University of Wisconsin School of Medicine and Public Health, Madison, WI 53792-4108, USA. ${ }^{2}$ Division of Interventional Radiology, Department of Radiology, University of Wisconsin School of Medicine and Public Health, Madison, WI 53792-4108, USA. ${ }^{3}$ Division of Pediatric Cardiology, Department of Pediatrics, University of Wisconsin School of Medicine and Public Health, 600 Highland Ave, Madison, WI 53792-4108, USA.

Received: 18 December 2017 Accepted: 4 April 2018

Published online: 17 April 2018

\section{References}

1. Chen E, Itkin M. Thoracic duct embolization for Chylous leaks. Semin Intervent Radiol. 2011;28:63-74.

2. Itkin M, Kucharaczuk JC, Kwak A, Trerotola SO, Kaiser LR. Nonoperative thoracic duct embolization for traumatic duct leak: experience in 109 patients. J Thorac Cardiovasc Surg. 2010;139:584-9.

3. Mery CM, Moffett BS, Khan MS, et al. Incidence and treatment of chylothorax after cardiac surgery in children: analysis of a large multiinstitution database. J Thorac Cardiovasc Surg. 2014;147:678-86.

4. Matsuo S, Takahashi G, Konishi A, Sai S. Management of refractory chylothorax after pediatric cardiovascular surgery. Pediatr Cardiol. 2013;34:1094-9.

5. Nath DS, Savla J, Khemani RG, Nussbaum DP, Greene CL, Wells WJ. Thoracic duct ligation for persistent chylothorax after pediatric cardiothoracic surgery. Ann Thorac Surg. 2009;88:246-51.

6. Savla JJ, Itkin M, Rossano JW, Dori Y. Post-operative chylothorax in patients with congenital heart disease. J Am Coll Cardiol. 2017;69:2410-22.

7. Dori Y, Keller MS, Rychik J, Itkin M. Successful treatment of plastic bronchitis by selective lymphatic embolization in a Fontan patient. Pediatrics. 2014; 134:e590-5.

8. Dori Y, Keller MS, Rome JJ, et al. Percutaneous lymphatic embolization of abnormal pulmonary lymphatic flow as treatment of plastic bronchitis in patients with congenital heart disease. Circulation. 2016;133:1160-70.

9. Dori Y, Keller MS, Fogel MA, Rome JJ, Whitehead KK, Harris MA, Itkin M. MRI of lymphatic abnormalities after functional single-ventricle palliation surgery. Am J Roentgenol. 2014:203:426-31.

10. Edwards JM, Kinmonth JB. Lymphovenous shunts in man. Br Med J. 1969;4: 579-81.

11. Aboul-Enein A, Eshmawy I, Arafa S, Abboud A. The role of lymphovenous communication in the development of postmastectomy lymphedema. Surgery. 1984;95(5):562-6.

12. Olszewski WL. Lymphovenous microsurgical shunts in treatment of lymphedema of the lower limbs: a 45-year experience of one surgeon/one center. Eur J Vasc Endovasc Surg. 2013;45(3):282-90.

\section{Ready to submit your research? Choose BMC and benefit from:}

- fast, convenient online submission

- thorough peer review by experienced researchers in your field

- rapid publication on acceptance

- support for research data, including large and complex data types

- gold Open Access which fosters wider collaboration and increased citations - maximum visibility for your research: over $100 \mathrm{M}$ website views per year 